\title{
Intraoperative fiducial-less patient registration using volumetric 3D ultrasound: a prospective series of 32 neurosurgical cases
}

\author{
Xiaoyao Fan, PhD, ${ }^{1}$ David W. Roberts, MD, ${ }^{2-4}$ Songbai Ji, DSc, ${ }^{1,2}$ Alex Hartov, PhD, ${ }^{1,3}$ and \\ Keith D. Paulsen, $\mathrm{PhD}^{1-3}$
}

${ }^{1}$ Thayer School of Engineering and ${ }^{2}$ Geisel School of Medicine, Dartmouth College, Hanover; and ${ }^{3}$ Norris Cotton Cancer Center and ${ }^{4}$ Section of Neurosurgery, Dartmouth-Hitchcock Medical Center, Lebanon, New Hampshire

\begin{abstract}
OBJECT Fiducial-based registration (FBR) is used widely for patient registration in image-guided neurosurgery. The authors of this study have developed an automatic fiducial-less registration (FLR) technique to find the patient-to-image transformation by directly registering 3D ultrasound (3DUS) with MR images without incorporating prior information. The purpose of the study was to evaluate the performance of the FLR technique when used prospectively in the operating room and to compare it with conventional FBR.
\end{abstract}

METHODS In 32 surgical patients who underwent conventional FBR, preoperative T1-weighted MR images (pMR) with attached fiducial markers were acquired prior to surgery. After craniotomy but before dural opening, a set of 3DUS images of the brain volume was acquired. A 2-step registration process was executed immediately after image acquisition: 1) the cortical surfaces from pMR and 3DUS were segmented, and a multistart sum-of-squared-intensity-difference registration was executed to find an initial alignment between down-sampled binary pMR and 3DUS volumes; and 2) the alignment was further refined by a mutual information-based registration between full-resolution grayscale pMR and 3DUS images, and a patient-to-image transformation was subsequently extracted.

RESULTS To assess the accuracy of the FLR technique, the following were quantified: 1) the fiducial distance error (FDE); and 2) the target registration error (TRE) at anterior commissure and posterior commissure locations; these were compared with conventional FBR. The results showed that although the average FDE $(6.42 \pm 2.05 \mathrm{~mm})$ was higher than the fiducial registration error (FRE) from FBR $(3.42 \pm 1.37 \mathrm{~mm})$, the overall TRE of FLR $(2.51 \pm 0.93 \mathrm{~mm})$ was lower than that of FBR $(5.48 \pm 1.81 \mathrm{~mm})$. The results agreed with the intent of the 2 registration techniques: FBR is designed to minimize the FRE, whereas FLR is designed to optimize feature alignment and hence minimize TRE. The overall computational cost of FLR was approximately 4-5 minutes and minimal user interaction was required.

CONCLUSIONS Because the FLR method directly registers 3DUS with MR by matching internal image features, it proved to be more accurate than FBR in terms of TRE in the 32 patients evaluated in this study. The overall efficiency of FLR in terms of the time and personnel involved is also improved relative to FBR in the operating room, and the method does not require additional image scans immediately prior to surgery. The performance of FLR and these results suggest potential for broad clinical application.

http://thejns.org/doi/abs/10.3171/2014.12.JNS141321

KEY WORDS patient registration; volumetric 3D ultrasound; 3DUS-MR registration; image-guided neurosurgery; diagnostic and operative techniques

$\mathrm{P}$ ATIENT registration based on preoperative images has become standard practice in open cranial neurosurgery to provide intraoperative navigation. It defines a transformation between the patient's head in the operating room (OR) and the preoperative (typically MR) im- ages, based on which surgical instruments (e.g., a stylus probe or a surgical microscope) can be optically tracked and coregistered with the MR images. The locations of these surgical tools and their orientations can then be displayed relative to the MR images in real time to provide

ABBREVIATIONS 3DUS = 3D ultrasound; $A C=$ anterior commissure; FBR = fiducial-based registration; FDE = fiducial distance error; FLR = fiducial-less registration; FRE = fiducial registration error; $\mathrm{OR}=$ operating room; $\mathrm{PC}=$ posterior commissure; $\mathrm{PMR}=$ preoperative MR images; $\mathrm{SBR}=$ surface-based registration; TRE = target registration error.

SUBMITTED July 2, 2014. ACCEPTED December 18, 2014.

INCLUDE WHEN CITING Published online July 3, 2015; DOI: 10.3171/2014.12.JNS141321.

DISCLOSURE This research was supported by National Institutes of Health Grant No. R01 CA159324-03. We acknowledge the support of Medtronic Navigation for use of the StealthStation S7, and Philips Healthcare for use of the iU22 ultrasound system. 
intraoperative navigational guidance for the surgeon. The accuracy of patient registration is, therefore, crucial to the surgical use of the navigation that is provided. The efficiency and robustness of the technique are equally important because they influence the workflow inside and outside the OR, as well as the associated health care costs.

Various methods have been developed to find an accurate patient-to-image transformation in neurosurgery, among which fiducial-based registration (FBR) is widely used for patient registration. Although skull-affixed fiducial markers provide higher accuracy (within $1 \mathrm{~mm}$ ), ${ }^{23}$ markers attached to the skin are typically used because they have practical convenience and acceptable accuracy. Specifically, a set of MR-compatible, skin-affixed fiducial markers (typically 10-12) is attached to the patient's scalp prior to MR acquisition. These markers are identified in the OR and matched with their counterparts in the preoperative MR images (pMR). However, FBR requires time and personnel resources, both before and at the time of surgery. Before surgery, personnel and expertise are needed 1) to attach the fiducial markers because their placement influences accuracy, ${ }^{30}$ and 2) to acquire the appropriate scan sequences with the markers attached (despite the availability of diagnostic scans previously acquired, which are usually available). At the time of surgery, personnel and expertise are needed to identify the markers with a digitizing stylus in the OR, and sometimes multiple repetitions are required to achieve an acceptable registration accuracy. The overall accuracy of FBR is typically $2-5 \mathrm{~mm}$ (as measured by fiducial registration error $[\mathrm{FRE}]^{8}$ ), but it can be compromised by marker movement (e.g., from skin movement). More importantly, because point-pair matching algorithms developed for FBR are designed to minimize the FRE, the target registration error (TRE) in the region of surgical interest, either on the cortical surface or deeper inside the brain, can be $\geq 5 \mathrm{~mm} .^{2,24}$

To improve the efficiency and accuracy of patient registration, techniques that do not use fiducial markers have been developed. For example, surface-based registration (SBR) matches digitized curvature of the head with its counterpart in the image scans instead of fiducial markers, with either a continuously tracked stylus probe ${ }^{9,13}$ or laser range scanning. ${ }^{28}$ The accuracy of extracranial SBR (based on external facial or forehead curvature) is reported to be comparable with FBR in terms of TRE. ${ }^{2}$ Although the accuracy can be further improved by intraoperative reregistration using the cortical surface (e.g., acquired by a laser range scanner ${ }^{2,24}$ or stereovision ${ }^{6}$ ) or the brain volume (e.g., acquired by ultrasound ${ }^{19}$ ), these intraoperative reregistration methods still require an initial alignment achieved by some form of patient registration, and thus they have not replaced patient registration.

$\mathrm{Ji}$ et al. reported an image-based fiducial-less registration (FLR) technique to register a true volumetric 3D ultrasound (3DUS) acquisition directly with the MR scans. This establishes the patient registration transformation between OR and image space without incorporating any prior information on the location of craniotomy, patient head position, or location and orientation of the US scan head in relation to the brain. ${ }^{17}$ The purpose of Ji et al.'s study was to develop and describe the overall technique, implement the associated algorithms and compare the FRE accuracy, and capture ranges and computational efficiencies of different approaches, e.g., using mutual information versus correlation ratio as the image similarity metric. Data from 10 surgical cases were evaluated but the FLRs were performed retrospectively, with a computational efficiency of 10-15 minutes, and the technique was not optimized for intraoperative use. In addition, the accuracy of the FLR was not fully evaluated; given the proof-of-principle nature of the study, only fiducial distance error (FDE) was reported. No effort was made to assess the TRE of the approach.

In this paper, we adopt $\mathrm{Ji}$ et al.'s technique, improve its computational efficiency, and evaluate it prospectively during neurosurgery by presenting results from 32 cases where the FLR was performed intraoperatively and immediately after craniotomy. To assess the accuracy of our method, we chose patients undergoing open cranial procedures with intraoperative image guidance based on FBR, and compared the standard FRE from FBR with FDE. We also quantified accuracy by independently locating internal targets that were present and identifiable in both MR and US images, and compared the TRE between FBR and our FLR technique.

\section{Methods \\ Surgical Cases and Procedure}

Thirty-two patients were evaluated intraoperatively at Dartmouth-Hitchcock Medical Center under a study protocol approved by the Institutional Review Board at Dartmouth. Informed consent was obtained from patients. Patient-recruitment criteria included: 1) the subject was scheduled for an open cranial procedure with FBR image guidance; 2) T1-weighted MR head scans with fiducial markers attached were available prior to surgery and used for FBR on a commercial navigation system; and 3) the planned craniotomy size was larger than or comparable in size with the 3DUS scan-head tip (dimension $2.5 \times 1.5$ $\mathrm{cm})$ so that the scan head could be held in firm contact with the dura mater to ensure image quality. Table 1 lists patients' age and sex, location and size of craniotomy, and type of lesion.

Prior to surgery, T1-weighted MR (with or without contrast enhancement) head scans were acquired (scan size $256 \times 256$ in-plane pixels, $120-144$ slices, voxel size $0.9375 \times 0.9375 \times 1.5 \mathrm{~mm}$ ). At the time of surgery, FBR was performed before craniotomy on a commercial navigation system, StealthStation S7 (Medtronic). A 3DUS system, iU22 (Philips Healthcare), with a broadband matrix array transducer (X3-1) was prepared in the OR for image acquisition. An active tracker was rigidly attached to the transducer, calibrated prior to surgery, ${ }^{11}$ and connected to the navigation system for optical tracking. After craniotomy but before dural opening, a set of 3DUS volumes was acquired. The acquisition range was set to its widest in the 2 angular directions $\left(-42.9^{\circ}\right.$ to $44.4^{\circ}$ in $\theta$ and $-36.6^{\circ}$ to $36.6^{\circ}$ in $\varphi$, respectively ${ }^{18}$ to cover the maximal brain volume allowed by the scan head, and the depth was set to capture the cortical surface opposite the craniotomy (approximately 15-20 cm). 
TABLE 1. Summary of data in 32 patients who underwent FLR

\begin{tabular}{|c|c|c|c|c|c|}
\hline Case No. & Sex & Age (yrs) & Lesion Location & Craniotomy Size (cm) & Lesion \\
\hline 1 & $\mathrm{~F}$ & 68 & Left frontal-temporal & $3.51 \times 3.13$ & Meningioma \\
\hline 2 & $\mathrm{~F}$ & 53 & Left temporal & $7.62 \times 5.61$ & Epilepsy \\
\hline 3 & M & 40 & Left frontal & $7.75 \times 5.71$ & Meningioma \\
\hline 4 & M & 58 & Left frontal & $7.64 \times 5.37$ & Aneurysm \\
\hline 5 & M & 68 & Left frontal & $3.74 \times 2.62$ & Meningioma \\
\hline 6 & $\mathrm{~F}$ & 57 & Right occipital & $5.18 \times 2.58$ & Meningioma \\
\hline 7 & $\mathrm{~F}$ & 59 & Left parietal & $3.98 \times 3.17$ & Meningioma \\
\hline 8 & M & 62 & Right frontal & $3.92 \times 3.63$ & Metastatic (primary melanoma) \\
\hline 9 & M & 44 & Right frontal & $3.28 \times 1.76$ & Cyst \\
\hline 10 & $\mathrm{~F}$ & 30 & Bilateral frontal & $3.07 \times 2.88$ & Colloid cyst \\
\hline 11 & $\mathrm{~F}$ & 75 & Left frontal & $7.33 \times 5.23$ & Meningioma \\
\hline 12 & M & 75 & Left frontal-temporal & $7.37 \times 4.59$ & Glioblastoma \\
\hline 13 & $\mathrm{~F}$ & 46 & Right parietal & $5.28 \times 4.79$ & Meningioma \\
\hline 14 & $\mathrm{~F}$ & 59 & Right frontal & $7.95 \times 5.85$ & Anaplastic mixed oligodendroglioma \\
\hline 15 & $\mathrm{~F}$ & 74 & Left frontal & $4.31 \times 2.38$ & Meningioma \\
\hline 16 & $\mathrm{~F}$ & 51 & Left frontal & $7.75 \times 5.41$ & Meningioma \\
\hline 17 & M & 60 & Left temporal & $4.58 \times 3.55$ & Glioblastoma \\
\hline 18 & M & 78 & Right occipital & $7.82 \times 5.84$ & Metastatic neuroendocrine carcinoma \\
\hline 19 & $\mathrm{~F}$ & 55 & Left occipital & $4.38 \times 1.98$ & Epilepsy \\
\hline 20 & M & 37 & Left frontal & $2.46 \times 1.69$ & Cyst \\
\hline 21 & $\mathrm{~F}$ & 65 & Bilateral frontal & $3.16 \times 2.28$ & Glioblastoma \\
\hline 22 & M & 66 & Left temporal & $3.28 \times 2.51$ & Gliosarcoma \\
\hline 23 & $\mathrm{~F}$ & 51 & Right frontal & $3.63 \times 2.65$ & Meningioma \\
\hline 24 & M & 83 & Right frontal-temporal & $4.36 \times 4.00$ & Glioblastoma \\
\hline 25 & $\mathrm{~F}$ & 61 & Right parietal & $7.32 \times 5.25$ & Meningioma \\
\hline 26 & M & 41 & Right temporal & $5.30 \times 4.95$ & Oligodendroglioma \\
\hline 27 & $\mathrm{~F}$ & 18 & Left frontal & $5.65 \times 4.23$ & Epilepsy \\
\hline 28 & $\mathrm{~F}$ & 63 & Right frontal & $2.51 \times 2.30$ & Meningioma \\
\hline 29 & $\mathrm{~F}$ & 68 & Right frontal & $5.77 \times 4.37$ & Metastatic carcinoma \\
\hline 30 & $\mathrm{~F}$ & 39 & Right frontal & $4.66 \times 4.50$ & Arteriovenous malformation \\
\hline 31 & M & 24 & Right frontal-parietal & $7.88 \times 5.71$ & Epilepsy \\
\hline 32 & M & 35 & Right frontal & $6.45 \times 3.49$ & Meningioma \\
\hline
\end{tabular}

\section{Two-Step Image Registration}

A 2-step image registration scheme was applied to find the fiducial-less US-to-MR transformation, the technical details of which have been previously reported ${ }^{17}$ and are briefly summarized here.

First, when pMR images became available (typically before the patient was brought into the OR), the brain was segmented with a level-set algorithm ${ }^{32}$ to estimate the shape of the cortical surface from the MR scans. To find an approximate initial alignment for subsequent registration, binary MR images were created and down-sampled by a factor of 4 (resulting MR volume size $56 \times 56$ in-plane pixels, 30-36 slices) to improve computational efficiency. A triangular mesh of the brain surface was subsequently generated based on the down-sampled binary images. Then, immediately after US acquisition, the 3DUS volume was rasterized into a cube $(50 \times 50 \times 50$ voxels $)$, and the cortical surface from the 3DUS volume was extracted by thresholding.
A multistart algorithm was executed to estimate the initial alignment between the down-sampled 3DUS and MR without any prior information on the location and/ or orientation of the US scan head in relation to the MR scans. Specifically, 100 nodal positions were randomly sampled from the triangular brain surface mesh to define starting locations for the scan head. The transducer was assumed to point perpendicularly to the brain surface. At each start location, 10 equally spaced orientations (from $0^{\circ}$ to $360^{\circ}$ ) were defined as the initial attitudes of the scan head, leading to a total of 1000 initial starting positions. A sum-of-squared-intensity-difference registration method was then applied to each initial position, and the one that produced the minimum sum-of-squared-intensity-difference (i.e., maximum similarity) was found. The resulting transformation, defined by the alignment between registered 3DUS and MR, was used as the starting position for a subsequent grayscale image registration to refine the alignment. 
The grayscale MR and 3DUS images were processed to enhance the similarity of their feature appearances (see reference 17 for image processing details), and a mutual information grayscale image registration was applied to refine the alignment between 3DUS and MR volumes, and create the final US-to-MR transformation, ${ }^{M R} T_{U S}$. The patient-to-image transformation was then computed as ${ }^{17}$

$$
\begin{aligned}
& { }^{M R} T_{\text {patient }}={ }^{M R} T_{U S} \times \operatorname{inv}\left({ }^{U S \text { tracker }} T_{U S}\right) \times{ }^{U S \text { tracker }} T_{\text {world }} \times \\
& \quad \operatorname{inv}\left(\text { patient } T_{\text {world }}\right),
\end{aligned}
$$

where ${ }^{U S \text { tracker }} T_{U S}$ was obtained from tracker calibration prior to surgery, and ${ }^{U S_{\text {tracker }}} T_{\text {world }}$ and ${ }^{\text {patient }} T_{\text {world }}$ were directly acquired from the tracking system and are transformations from the world coordinates to the tracked ultrasound scan head and the patient, respectively.

Figure 1 illustrates binary (Fig. 1A) and grayscale registrations (before [Fig. 1B] and after [Fig. 1C]) from Cases 1,16 , and 24 (upper to lower rows, respectively). In each column, the US images were resampled at the MR voxel locations based on the US-to-MR transformation.

\section{Data Analysis}

As a quantitative assessment of the accuracy of our 3DUS FLR, we first compared the standard FRE from the FBR with the FDE from our approach. Specifically, for each case, a patient-to-image transformation, $T_{F B R}$; the locations of the fiducial markers digitized in OR space, $q_{i}$; and their counterparts in image space, $p_{i}$; were directly obtained from the commercial navigation system where FBR was performed, and FRE (not normally provided on the navigation system) was computed as the root mean square error ${ }^{12}$

$$
F R E=\sqrt{\frac{1}{M} \sum_{i=1}^{M}\left\|q_{i}-T_{F B R} p_{i}\right\|^{2}}
$$

and similarly, FDE was computed as

$$
F D E=\sqrt{\frac{1}{M} \sum_{i=1}^{M}\left\|q_{i}-T_{F L R} p_{i}\right\|^{2}}
$$

where $T_{F L R}$ refers to ${ }^{M R} T_{\text {patien }}$, the patient-to-image transformation obtained from the FLR.

As a second accuracy assessment, we compared the TRE of internal features between FBR and FLR. We chose the anterior commissure (AC) and posterior commissure (PC) as true soft-tissue targets because they can be accurately localized in both MR and 3DUS as voxels (points). Specifically, for each patient, when AC and/or PC were identifiable in both MR and 3DUS images, they were located independently by the neurosurgeon (D.W.R.), as shown in Fig. 2. Because of the difficulties in interpreting raw or rasterized 3DUS image volumes without any prior information on their orientation or location of acquisition, we transformed the 3DUS volume into the MR image space using the FBR transformation to assist the US interpretation, and the 3DUS volume was resampled at the MR voxel locations. However, when identifying the actual targets (i.e., AC and PC), the 3DUS images were shown to the neurosurgeon without the MR overlay. AC and PC were each identified independently as voxels having coordinates $p(x, y, z)$ in the US image volume and $q(x, y, z)$ in the MR image volume.

TRE at the target (i.e., $\mathrm{AC}$ and PC) was computed $\mathrm{as}^{8}$

$$
T R E=\|q-T \cdot p\|
$$

and overall TRE was found as

$$
T R E=\sqrt{\frac{1}{N} \sum_{i=1}^{N}\left\|q_{i}-T \cdot p_{i}\right\|^{2}}
$$

where $T$ represents the US-to-MR transformation obtained from FBR $\left(T_{F B R}\right)$ or FLR $\left(T_{F L R}\right)$, and $\mathrm{N}$ is the total number of targets.

To eliminate the possibility that misalignment between 3DUS and pMR was due to inaccurate US image calibration, we calculated the difference between transformations from FBR and FLR. Specifically, we found the transformation between $T_{F B R}$ and $T_{F L R}$, decomposed it into 3 translational and 3 rotational (6 degrees of freedom) components, and transformed these elements into US tracker space. If the calibration was inaccurate, a consistent shift and/or rotation would exist in all 32 patients, and the mean value of the 3 translational and 3 rotational components would not be near 0 .

\section{Results}

\section{FRE and FDE Evaluation}

To quantitatively evaluate the registration accuracy of FLR, we report the FDE and compare these results with FRE (from FBR) for the 32 patients in Fig. 3. The fiducial-less FDE (mean, red crosses) and fiducial-based FRE (mean, blue empty circles) from each patient case are plotted (left), and the overall FRE and FDE are compared in a boxplot (right). The results show that the overall fiducialless FDE $(6.42 \pm 2.05 \mathrm{~mm})$ is significantly larger than the fiducial-based FRE $(3.42 \pm 1.37 \mathrm{~mm})$. Only 1 patient (Case 11) showed a FDE lower than FRE.

Although the overall FDE is higher than the FRE, the FLR results are visually more accurate when overlaying the 3DUS with MR. For example, Fig. 4 shows an overlay of MR (red) and US (green) from Cases 1, 16, and 24 (the same cases in Fig. 1) using FLR (upper) and FBR (lower), respectively. As indicated in the figure, internal features such as ventricles and tumor boundaries (see white arrows) are accurately aligned using FLR, whereas FBR exhibits misalignment that is visually evident. 


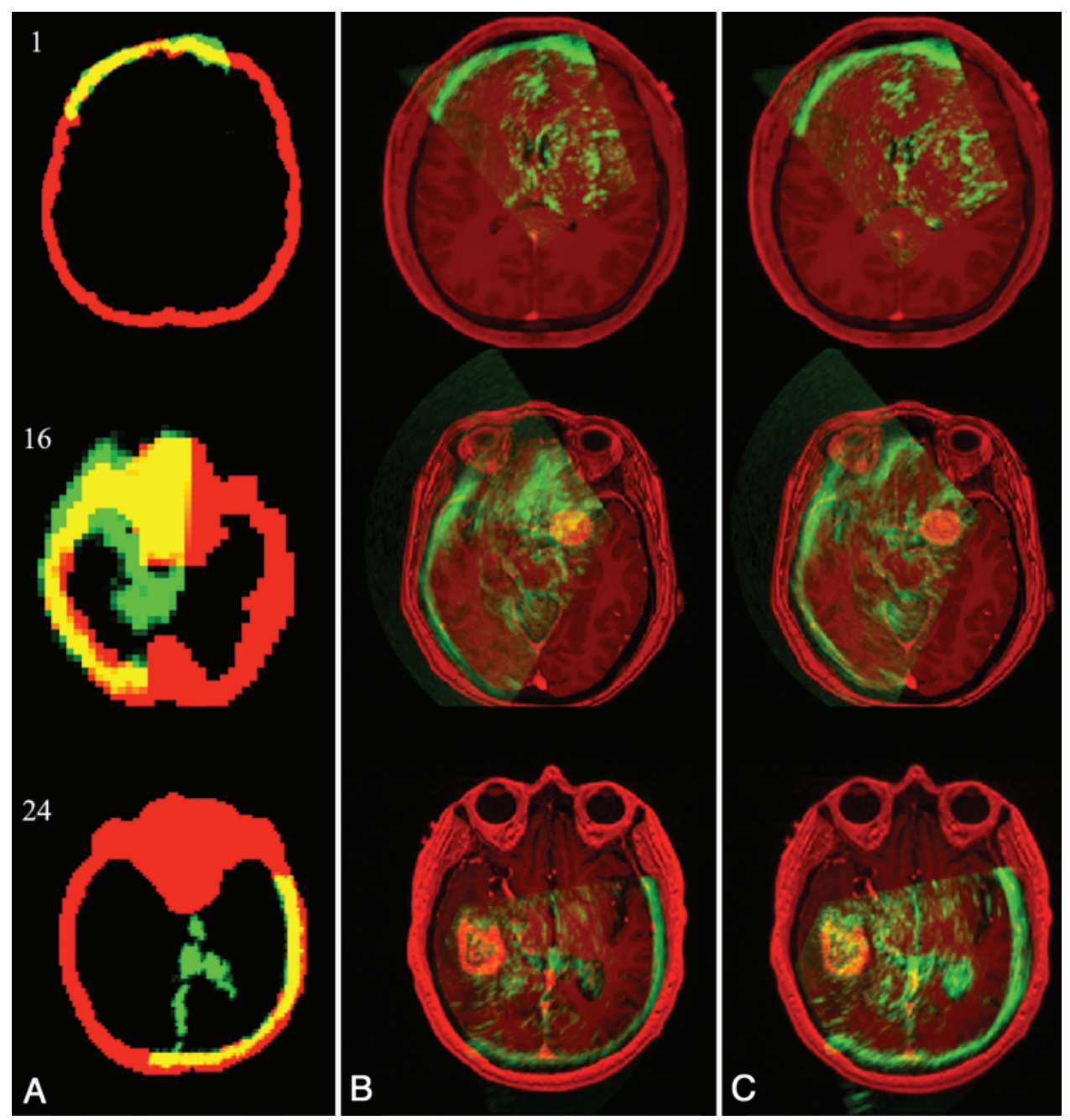

FIG. 1. FLRs from Cases 1, 16, and 24. A: Binary registration of cortical surfaces from down-sampled MR (red) and resliced US (green). B: Overlay of MR and US after binary registration. C: Overlay of MR and US after grayscale registration. Figure is available in color online only.

\section{TRE Evaluation}

As a second accuracy assessment, we report the TRE of $\mathrm{AC}$ and $\mathrm{PC}$ for all 32 patients and compare TREs obtained from FLR and FBR in Fig. 5. Due to significant brain deformation caused by lesion and intracranial pressure, the AC was not identifiable in the US or MR in Cases 4, 10, $18,19,29$, and 32 , and the PC was not identifiable in Cases 14, 29, and 32. These data are not included in Fig. 5.

The results show that the TREs of FLR at AC (2.62 \pm $1.11 \mathrm{~mm}), \mathrm{PC}(2.38 \pm 1.15 \mathrm{~mm})$, and the overall TRE $(2.51$ $\pm 0.93 \mathrm{~mm})$ are significantly lower than those of FBR $(5.54 \pm 1.92 \mathrm{~mm}, 5.41 \pm 1.93 \mathrm{~mm}$, and $5.48 \pm 1.81 \mathrm{~mm}$ for AC, PC, and overall TRE, respectively). The overall TRE of FLR was also lower than that of FBR in every case.

We report the difference between transformations from FBR and FLR to show that the US image calibration error was minimal in this study. Our results produced an average near 0 in all 6 degrees of freedom $(-0.17 \pm 2.54 \mathrm{~mm}$, $0.14 \pm 2.90 \mathrm{~mm}$, and $0.28 \pm 2.86 \mathrm{~mm}$ in translational directions, and $0.39^{\circ} \pm 2.16^{\circ},-0.13^{\circ} \pm 2.36^{\circ}, 0.62^{\circ} \pm 1.55^{\circ}$ in rotational directions, respectively), indicating that a con- sistent calibration error did not exist. In addition, we purposely acquired other 3DUS images with different view angles at the time of acquisition, overlaid those images with pMR using $T_{F L R}$, and visually assessed the alignment. Figure 6 shows an example from the patient in Case 1, where image A was used for registration to obtain $T_{F L R}$ and images $\mathrm{B}$ and $\mathrm{C}$ were acquired with different scanhead positions (from the same craniotomy) immediately after acquisition of image A. All 3 images were overlaid with pMR using the same transformation. Although the image quality appears to vary slightly between acquisitions, features are well aligned (e.g., see white arrows) in all 3 cases, indicating that the influence of US image calibration and acquisition/tracking errors was minimal.

\section{Computational Efficiency}

The computational efficiency was evaluated on a Linux computer $(2.33 \mathrm{GHz}, 16 \mathrm{G}$ RAM) in MATLAB (MATLAB R2012b, MathWorks). Segmentation was performed prior to craniotomy, usually as soon as the pMR images became available. The automatic level-set segmentation 

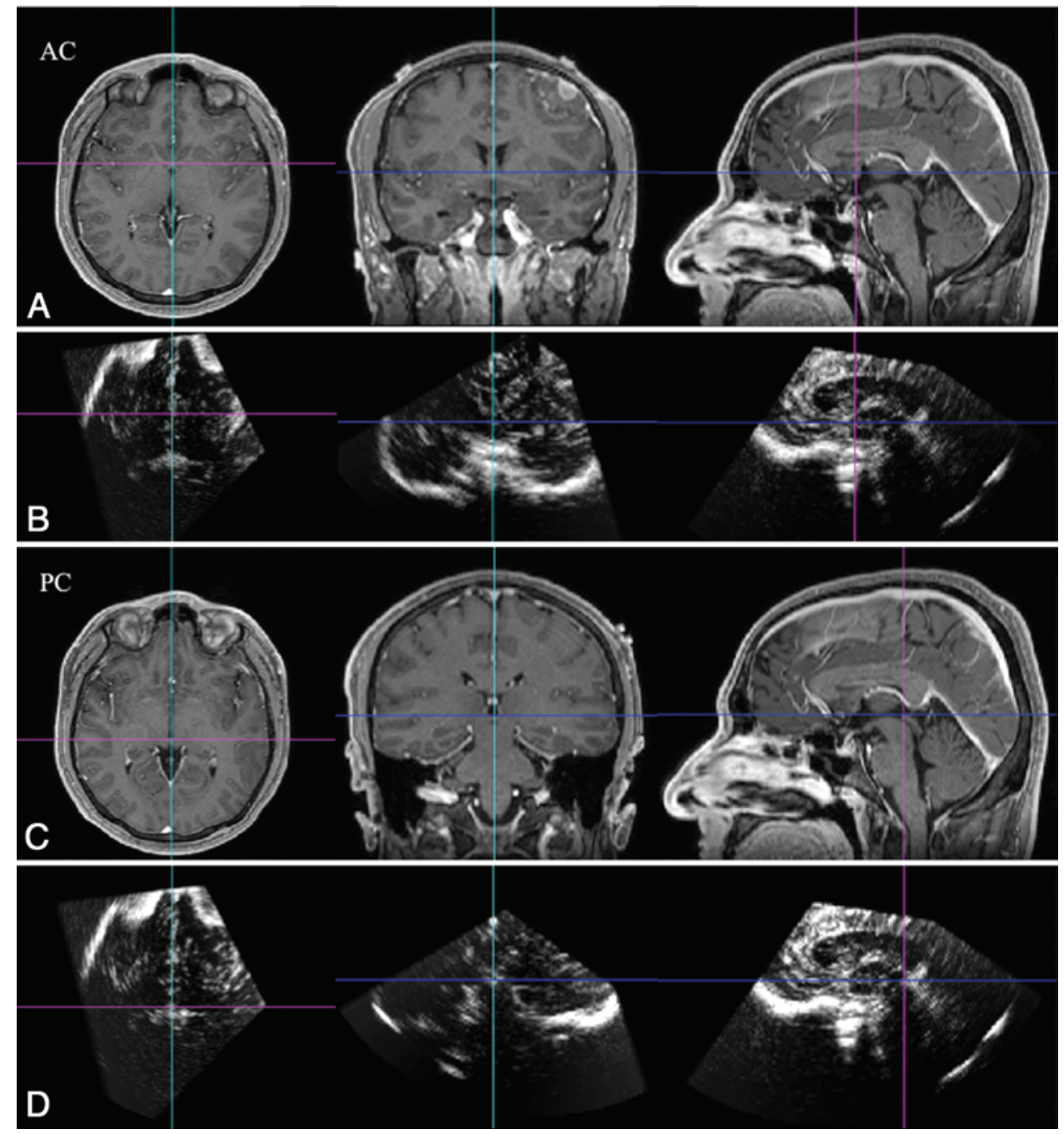

FIG. 2. Identification of $A C(A$ and $B)$ and $P C(C$ and $D)$ in MR and 3DUS independently. Magenta, blue, and cyan lines represent the coronal, axial, and sagittal cross-sections at the point of interest, respectively. Figure is available in color online only.

was typically finished within 2 minutes to provide an outer shape of the cortical surface that was sufficiently accurate for binary registration. Parallel computing was used for the binary registration step, where 1000 multistart registrations were sent to $248 \mathrm{CPUs}$, and the total computational time was typically 2-3 minutes. The mutual information grayscale image registration was typically finished within 2 minutes using 12 CPUs, but the actual computational cost depended on the initial alignment (i.e., the results from binary registration) and ranged from 1 to 4 minutes. Thus, the total computational time to complete a US-to-MR FLR (which required minimal user interaction) was typically less than 5 minutes after the 3DUS image became available.

\section{Discussion}

In this study, we applied FLR intraoperatively during 32 open cranial surgeries to find a transformation between physical space in the OR and image space defined by preoperative MR. Our technique established a patient registration with an FRE equivalent to that of FBR and a
TRE superior to that of FBR without using fiducial markers. Hence, our technique can be executed more efficiently and cost-effectively in terms of the personnel and time involved to complete the patient-registration procedure (less than 10 minutes for FLR or less than what is commonly taken by FBR).

We evaluated the performance of FLR in terms of accuracy and efficiency. We quantified FLR accuracy first by measuring its FDE compared with FRE from FBR performed on a commercial navigation system. Although the more extensive results presented here differ from earlier findings that showed no significant difference between FDE and FRE, we found that the internal features registered by FLR in the 3DUS matched better visually with pMR than those from FBR. Not surprisingly, FRE was lower than FDE because FBR is explicitly designed to minimize distances between fiducial markers and their appearance in image space. However, clinically, these markers may shift from their original positions (when pMR was acquired), e.g., in lateral and/or normal directions, due to skin movement when a stylus probe is placed in contact with the skin to digitize them. Algorithms that minimize 

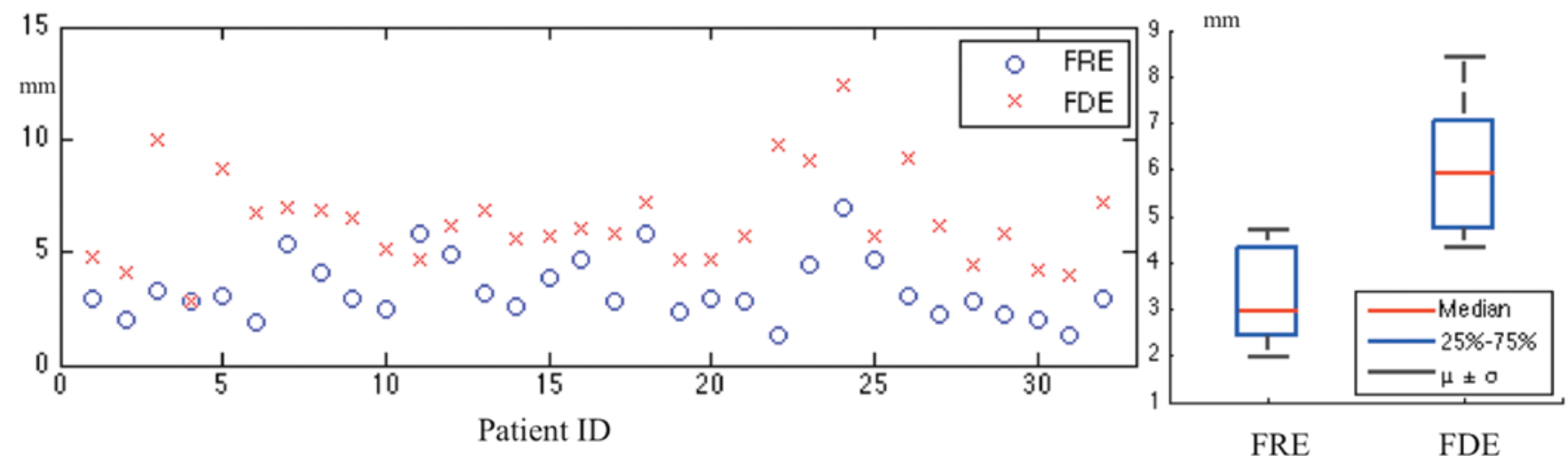

FIG. 3. Comparison of fiducial-less FDE (mean) with fiducial-based FRE (mean) for the 32 patients. The mean FDE (red crosses) and FRE (blue empty circles) for each patient are plotted (left), and a boxplot of these data is shown (right). Figure is available in color online only.

FRE typically do not account for such errors. As a result, the accuracy of image guidance with FBR may not be well represented by FRE, and probably decreases further when the surgical target is deeper in the brain and far away from the surface fiducial markers. In fact, in all 32 patients we observed visually identifiable misalignment between 3DUS and pMR (e.g., Fig. 4) from FBR, whereas the features were well aligned by FLR.

To assess accuracy within the brain, we chose 2 softtissue targets and compared FLR with FBR. The results show that FLR is significantly more accurate than FBR in terms of TRE. Although anatomical structures have been used for TRE assessment in the literature, we picked the $\mathrm{AC}$ and PC because they are features deep in the brain, and can be identified and located in US and MR independently and accurately as homologous points, whereas other types of targets may introduce additional errors in the accuracy assessment. For example, features such as ventricles and falx require segmentation in both MR and 3DUS, and involve additional algorithms such as iterative closest point distance to calculate distances between objects.

Existing patient registration techniques, including FBR and SBR, typically provide a TRE of approximately 3-4 $\mathrm{mm}$ and $\geq 5 \mathrm{~mm},{ }^{22}$ according to the literature. The increase in registration accuracy in terms of TRE from 5.48 $\mathrm{mm}$ (FBR) to $2.51 \mathrm{~mm}$ (FLR) is significant in intracranial neurosurgical procedures. The accuracy of image guidance is directly related to the resulting surgical outcomes,

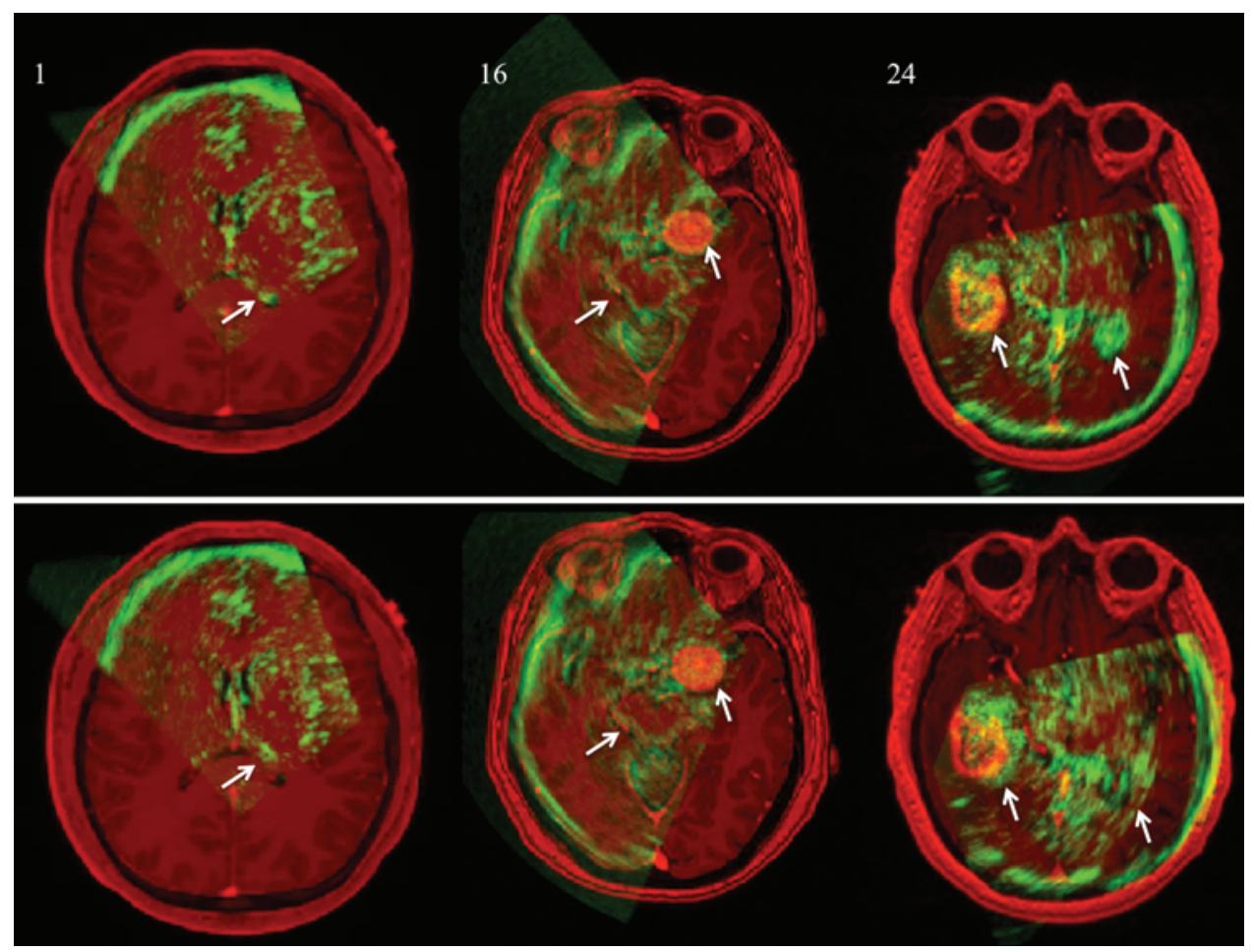

FIG. 4. Visual comparison of FLR (upper row) and FBR (lower row) for Cases 1, 16, and 24 by overlaying US (green) with MR (red) images. White arrows point to examples of internal features that are well aligned with FLR but misaligned with FBR. Figure is available in color online only. 

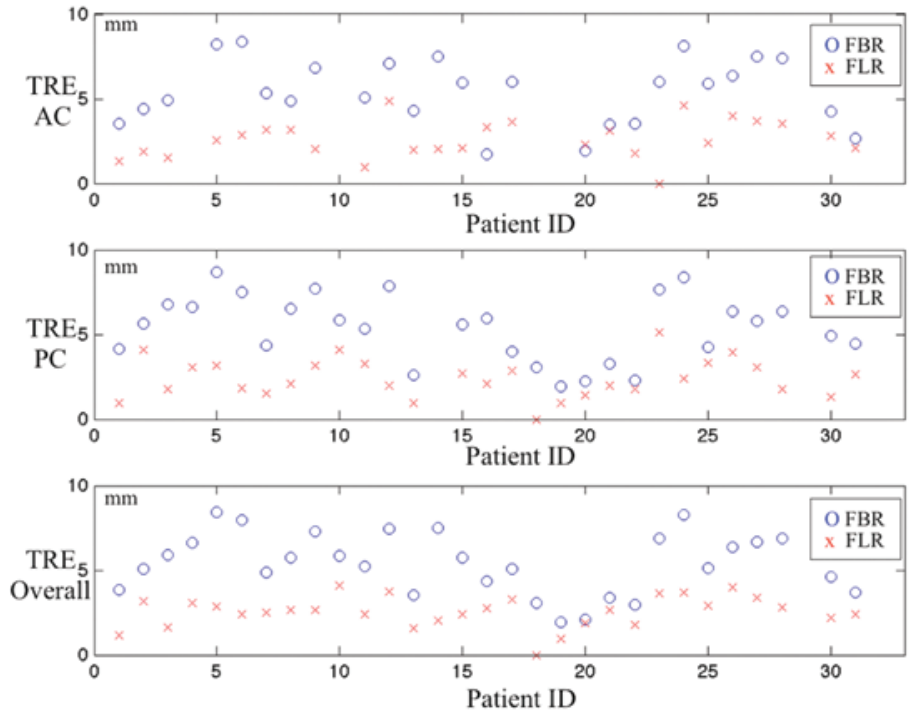

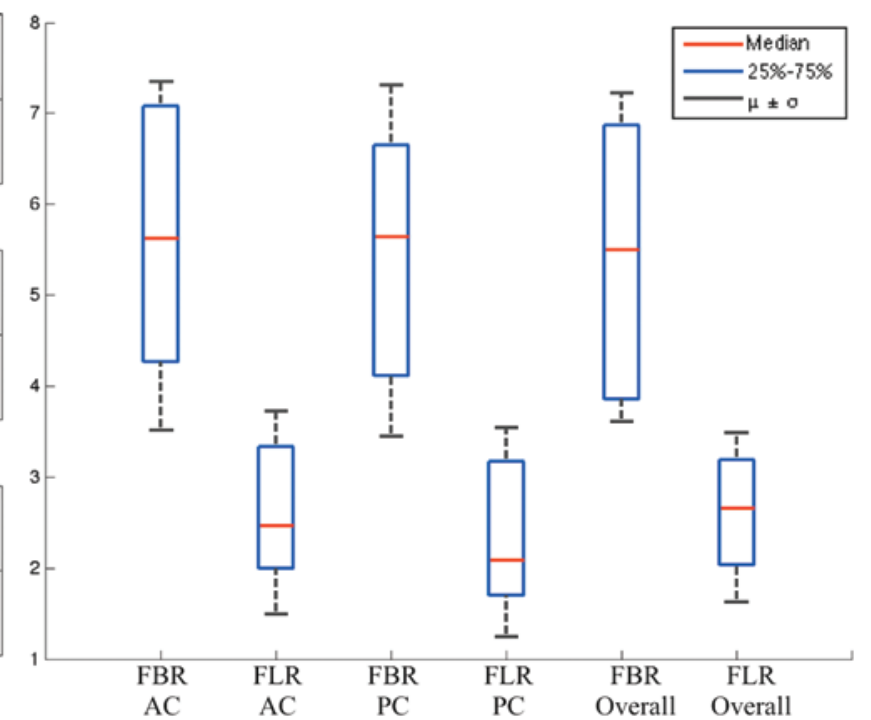

FIG. 5. Comparison of TRE from FBR and FLR of the AC and PC for the 32 cases. Left: Image showing 3 plots-the TRE at AC, $\mathrm{PC}$, and the overall TRE that combines both targets, respectively, where blue empty circles represent data from FBR, and red crosses represent data from FLR. Right: The corresponding boxplot. Figure is available in color online only.

and ultimately to patient survival. ${ }^{1,20}$ For example, in a meta-analysis of 416 patients with glioblastoma multiforme, ${ }^{20}$ a significant survival advantage was associated with resection of $\geq 98 \%$ of tumor volume. Single-institution data support the premise that intraoperative image guidance increases the completeness of resection, although the impact on survival that is directly attributable to the availability of image guidance is less certain.,31 The increase in TRE accuracy is also of significant value when certain functions of the brain are involved in or near the surgical site. The accuracy of image guidance often degrades over time during surgery due to brain shift. Various techniques have been developed to account for the brain deformation resulting from surgical events such as dural opening, resection, and retraction. $3,5,7,10,14,15,21,25-27$ Nevertheless, all of these compensation methods depend on an initial alignment achieved by patient registration; hence, the registration accuracy attained later in a case is directly influenced by the accuracy of the initial patient registration.

Our FLR technique provides significant time and cost advantages over FBR. First, preoperative MR sequences with markers attached are not required immediately pri- or to surgery, because diagnostic MR scans previously acquired (which are typically available) can be directly used for FLR. Hence no additional personnel and expertise are needed to attach the fiducial markers and acquire MR scans, resulting in significant time (up to 1 hour) and cost efficiencies (i.e., fewer MR scans). Instead, a 3DUS system is used to obtain real-time images with acquisition times and costs that are significantly less than MR scans. At the time of surgery, FBR requires personnel and expertise to identify the markers with a digitizing stylus in the OR. Depending on the navigation platform, fiducial markers in the MR images may need to be identified manually and multiple repetitions may be required to achieve an acceptable registration accuracy, further increasing the time involved. In comparison, the total time involved for FLR was less than 10 minutes, and about half of this time was associated with the automated computational processing of the image data. Specifically, after craniotomy, 3DUS acquisition introduced minimal interruption to surgical flow and was usually finished within 5 minutes, including the time for draping the scan head, manipulating the scan head, and fine-tuning acquisition parameters to ensure US
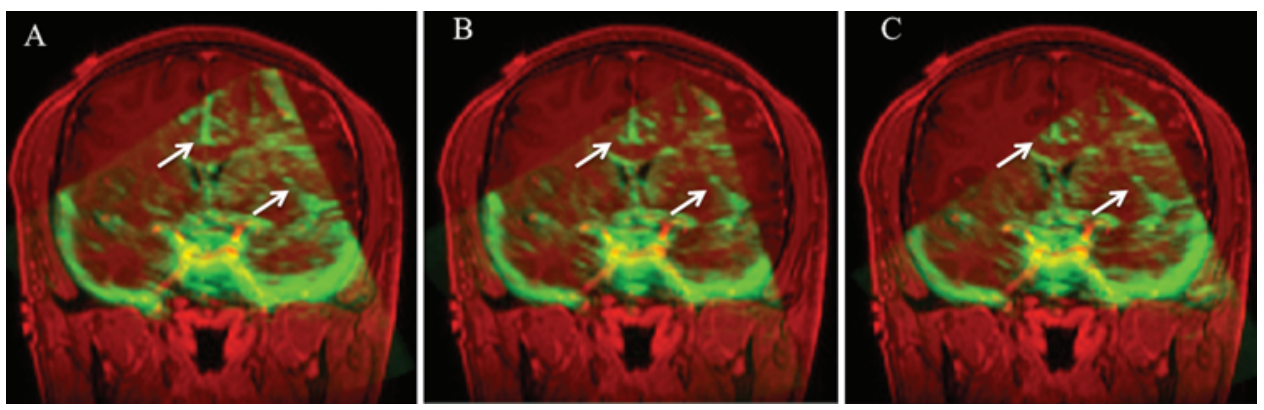

FIG. 6. Example of visual assessment of accuracy by overlaying pMR (red) with different 3DUS images (green). FLR was performed based on panel $\mathbf{A}$, and panels $\mathbf{B}$ and $\mathbf{C}$ were acquired immediately after $\mathbf{A}$ but with different scan-head positions. White arrows designate features that are well aligned in all 3 US acquisitions coregistered with FLR based only on panel A. Figure is available in color online only. 
image quality. The total computational time to complete a US-to-MR FLR, including the multistart binary registration (approximately 2-3 min) and a second grayscale registration (approximately $2 \mathrm{~min}$ ), was typically less than 4-5 minutes. These computations do not directly alter or influence surgical flow, as the algorithms are completely automatic and the process requires minimal user interaction and can be executed while the surgeon is opening the dura. Thus, the overall efficiency of FLR in the OR exceeds FBR, and the approach certainly requires less labor and fewer personnel.

The registration algorithms to align 3DUS and MR images were implemented in the Insight Segmentation and Registration Toolkit, which is an open-source platform and is free of cost. Other image-processing work was implemented in MATLAB, for which the cost varies depending on licensing (ranging from free to approximately $\$ 2000)$. However, these tasks can be implemented in $\mathrm{C}$ or other programming languages and packaged for clinical settings. Hence, the overall effort and resources required to implement FLR in the OR are very favorable. In principle, no barriers exist to incorporating the approach within a commercially available navigation platform such as the StealthStation.

Surface-based registration (SBR) is another patient registration technique that does not require fiducials and has been developed and implemented in some commercial navigation platforms. SBR matches digitized curvature of the head with its counterpart in the image scans instead of fiducial markers, with either a continuously tracked stylus probe ${ }^{9,13}$ or laser range scanning. ${ }^{28}$ Similar to FLR, SBR does not require additional preoperative MR scans with fiducials, and is especially valuable when fiducial markers are not available (e.g., in emergency cases). However, similar to FBR, SBR matches the exterior features and thus is prone to errors from skin movement or variability in probe contact and/or patient position. Compared with SBR, FLR provides similar time savings and cost advantages but minimizes misalignment of internal features, hence improving TRE in areas of the brain that are often of the highest surgical interest.

In this study, we evaluated FLR prospectively in 32 cases with craniotomies in different locations. These included the frontal, temporal, parietal, and occipital lobes, indicating the robustness of the approach. We showed that FLR does not depend on craniotomy location per se, because image features are used for registration and are generally visible in the 3DUS regardless of craniotomy location (although a craniotomy size large enough to accommodate the US scan head is required). Coincidently, no posterior fossa surgical cases occurred in the enrollment group; this location was not an exclusion criterion. The feasibility of FLR is expected to be the same in the posterior fossa as long as image features are visible in 3DUS. We also did not limit the FLR search range (i.e., specify the initial starting position for the binary registration). Three cases involving different regions of interest were chosen for illustration purposes (Figs. 1 and 4). A total of 100 starting positions were randomly sampled around the cortical surface, each with 10 rotation angles, resulting in the completion of 1000 binary registrations in a total computational cost of
2-3 minutes. In future applications, the efficiency of the FLR technique evaluated in this study could be improved by limiting the search range (i.e., number of starting positions) of the multistart binary registration. For example, the brain could be divided into zones that represent different lobes, and the search range could be limited to the lobe of interest. As a result, the computational time could be decreased by an additional 1-2 minutes to improve the time efficiency of FLR even further.

Because the binary registration relies on the segmented cortical surface for image features, 3DUS was acquired with the widest angular settings and appropriate depth to obtain maximum cortical surface on the side opposite to the craniotomy. In addition, the surgeon manipulated the scan head to cover the maximum brain volume in order to capture sufficient features for the binary and grayscale registrations. Alternatively, multiple scans could be acquired and combined to form a larger brain volume, ${ }^{16}$ which may further improve the efficiency and robustness of our approach.

In this study, we used a commercial navigation system, StealthStation S7 (Medtronic), for optical tracking. We compared the performance of FLR with that of FBR performed on the StealthStation. However, our FLR approach does not depend on the navigation platform. Indeed, in the prior study by Ji et al., a customized optical tracking system, Polaris (The Northern Digital Inc.), was used instead of a StealthStation.

Some limitations are evident in the FLR method evaluated here. First, a 3DUS image needs to be acquired before the dura is opened to minimize the influence of brain shift. After dural opening, nonrigid brain deformation $\geq 1 \mathrm{~cm}^{29}$ can occur, both on the cortical surface and deep in the brain. Therefore, a direct rigid registration between 3DUS and pMR may no longer be accurate, whereas a direct nonrigid registration between 3DUS and MR that can compensate for brain deformation is likely to be challenging to implement because of the different imaging modalities.

Second, the technique is designed to directly register 3DUS with T1-weighted MR images, but not other image sequences or modalities. The parameters in the technique described in this study, including for segmentation and image processing, were optimized to enhance common features between 3DUS and T1-weighted MR images. However, the registration algorithms, including both binary and grayscale registrations, do not depend on the imaging modality per se. Therefore, with necessary changes in segmentation and image processing, our technique could potentially be applied to image data from CT scans (or other MR sequences) where internal features are visible.

Third, a craniotomy of a size larger than the tip of the 3DUS scan head is required to ensure image-acquisition quality. In this study, we used a transducer with a tip size of $2.5 \times 1.5 \mathrm{~cm}$, and patient recruitment was limited by the intended craniotomy size. As a result, this FLR method is not possible in neurosurgical procedures such as biopsy through a bur hole (at least with the transducer head used in this study). In addition, at this point, a customized patient registration could not be uploaded to the commercial navigation platform (i.e., StealthStation S7), and patient registration achieved via the navigation platform could 
not be modified. In this study, we displayed FLR views through a customized graphical user interface on another workstation that mimicked the StealthStation. This required additional software (StealthLink, Medtronic) to retrieve real-time tracking information on the instruments used in surgery from the StealthStation. However, all technical procedures and software described in this study could be integrated into a single navigation platform in the future. While the limitations summarized here may preclude the FLR technique from broad clinical application under its current implementation, we have shown that it provides improved TRE with cost and time efficiencies over FBR, suggesting its potential for use in image-guided neurosurgical procedures in the future.

\section{Conclusions}

We have developed a 3DUS-based FLR to find an accurate patient-to-image transformation for intraoperative image guidance. We applied the approach prospectively during 32 clinical cases in the OR, all of which were successfully registered intraoperatively and immediately after craniotomy. In addition to visual validation, we quantified the accuracy of our approach by comparing the FDE with the FRE from FBR and chose AC and PC as targets to assess TRE. Our results show that although the overall FDE $(6.42 \pm 2.05 \mathrm{~mm})$ is higher than the FRE from FBR (3.42 $\pm 1.37 \mathrm{~mm})$, the average TRE $(2.51 \pm 0.93 \mathrm{~mm})$ was lower than that of FBR $(5.48 \pm 1.81 \mathrm{~mm})$. The computational cost was approximately $4-5$ minutes using parallel computing. All computations were executed with minimal user interaction while the surgeon was opening the dura. Hence, the procedure did not interrupt surgical flow. The overall efficiency in terms of the time and personnel involved is improved relative to FBR in the OR, and the method does not require additional image scans prior to surgery. These results suggest the potential for intraoperative FLR in neurosurgical procedures.

\section{References}

1. Buckner JC: Factors influencing survival in high-grade gliomas. Semin Oncol 30 (6 Suppl 19):10-14, 2003

2. Cao A, Thompson RC, Dumpuri P, Dawant BM, Galloway RL, Ding S, et al: Laser range scanning for image-guided neurosurgery: investigation of image-to-physical space registrations. Med Phys 35:1593-1605, 2008

3. Carter TJ, Sermesant M, Cash DM, Barratt DC, Tanner C, Hawkes DJ: Application of soft tissue modelling to imageguided surgery. Med Eng Phys 27:893-909, 2005

4. Claus EB, Horlacher A, Hsu L, Schwartz RB, Dello-Iacono D, Talos F, et al: Survival rates in patients with low-grade glioma after intraoperative magnetic resonance image guidance. Cancer 103:1227-1233, 2005

5. Fan X, Ji S, Fontaine K, Hartov A, Roberts D, Paulsen K: Simulation of brain tumor resection in image-guided neurosurgery. Proc SPIE 7964:79640U, 2011

6. Fan X, Ji S, Hartov A, Roberts DW, Paulsen KD: Stereovision to MR image registration for cortical surface displacement mapping to enhance image-guided neurosurgery. Med Phys 41:102302, 2014

7. Ferrant M, Nabavi A, Macq B, Black PM, Jolesz FA, Kikinis $\mathrm{R}$, et al: Serial registration of intraoperative MR images of the brain. Med Image Anal 6:337-359, 2002

8. Fitzpatrick JM, West JB, Maurer CR Jr: Predicting error in rigid-body point-based registration. IEEE Trans Med Imaging 17:694-702, 1998

9. Friets EM, Strohbehn JW, Roberts DW: Curvature-based nonfiducial registration for the Frameless Stereotactic Operating Microscope. IEEE Trans Biomed Eng 42:867-878, 1995

10. Garlapati RR, Roy A, Joldes GR, Wittek A, Mostayed A, Doyle B, et al: More accurate neuronavigation data provided by biomechanical modeling instead of rigid registration. $\mathbf{J}$ Neurosurg 120:1477-1483, 2014

11. Hartov A, Paulsen K, Ji S, Fontaine K, Furon ML, Borsic A, et al: Adaptive spatial calibration of a 3D ultrasound system. Med Phys 37:2121-2130, 2010

12. Helm PA, Eckel TS: Accuracy of registration methods in frameless stereotaxis. Comput Aided Surg 3:51-56, 1998

13. Henderson JM, Smith KR, Bucholz RD: An accurate and ergonomic method of registration for image-guided neurosurgery. Comput Med Imaging Graph 18:273-277, 1994

14. Ji S, Fan X, Hartov A, Roberts D, Paulsen K: Estimation of Intraoperative Brain Deformation, in Payan Y (ed): Soft Tissue Biomechanical Modeling for Computer Assisted Surgery. Berlin: Springer, 2012, Vol 11, pp 97-133

15. Ji S, Hartov A, Roberts D, Paulsen K: Data assimilation using a gradient descent method for estimation of intraoperative brain deformation. Med Image Anal 13:744-756, 2009

16. Ji S, Roberts DW, Hartov A, Paulsen KD: Combining multiple true 3D ultrasound image volumes through re-registration and rasterization. Med Image Comput Comput Assist Interv 12:795-802, 2009

17. Ji S, Roberts DW, Hartov A, Paulsen KD: Intraoperative patient registration using volumetric true 3D ultrasound without fiducials. Med Phys 39:7540-7552, 2012

18. Ji S, Roberts DW, Hartov A, Paulsen KD: Real-time interpolation for true 3-dimensional ultrasound image volumes. J Ultrasound Med 30:243-252, 2011

19. Ji S, Wu Z, Hartov A, Roberts DW, Paulsen KD: Mutualinformation-based image to patient re-registration using intraoperative ultrasound in image-guided neurosurgery. Med Phys 35:4612-4624, 2008

20. Lacroix M, Abi-Said D, Fourney DR, Gokaslan ZL, Shi W, DeMonte F, et al: A multivariate analysis of 416 patients with glioblastoma multiforme: prognosis, extent of resection, and survival. J Neurosurg 95:190-198, 2001

21. Lunn KE, Paulsen KD, Lynch DR, Roberts DW, Kennedy FE, Hartov A: Assimilating intraoperative data with brain shift modeling using the adjoint equations. Med Image Anal 9:281-293, 2005

22. Mascott CR, Sol JC, Bousquet P, Lagarrigue J, Lazorthes Y, Lauwers-Cances V: Quantification of true in vivo (application) accuracy in cranial image-guided surgery: influence of mode of patient registration. Neurosurgery 59:ONS146ONS156, 2006

23. Maurer CR Jr, Fitzpatrick JM, Wang MY, Galloway RL Jr, Maciunas RJ, Allen GS: Registration of head volume images using implantable fiducial markers. IEEE Trans Med Imaging 16:447-462, 1997

24. Miga MI, Sinha TK, Cash DM, Galloway RL, Weil RJ: Cortical surface registration for image-guided neurosurgery using laser-range scanning. IEEE Trans Med Imaging 22:973-985, 2003

25. Miga MI, Staubert A, Paulsen KD, Kennedy FE, Tronnier VM, Roberts DW, et al: Model-updated image-guided neurosurgery: Preliminary analysis using intraoperative MR. Med Image Comput Comput Assist Interv 1935:115-124, 2000

26. Mostayed A, Garlapati RR, Joldes GR, Wittek A, Roy A, Kikinis R, et al: Biomechanical model as a registration tool for image-guided neurosurgery: evaluation against BSpline registration. Ann Biomed Eng 41:2409-2425, 2013

27. Paulsen KD, Miga MI, Kennedy FE, Hoopes PJ, Hartov A, 
Roberts DW: A computational model for tracking subsurface tissue deformation during stereotactic neurosurgery. IEEE

Trans Biomed Eng 46:213-225, 1999

28. Raabe A, Krishnan R, Wolff R, Hermann E, Zimmermann $\mathrm{M}$, Seifert V: Laser surface scanning for patient registration in intracranial image-guided surgery. Neurosurgery 50:797803, 2002

29. Roberts DW, Hartov A, Kennedy FE, Miga MI, Paulsen KD: Intraoperative brain shift and deformation: a quantitative analysis of cortical displacement in 28 cases. Neurosurgery 43:749-760, 1998

30. West JB, Fitzpatrick JM, Toms SA, Maurer CR Jr, Maciunas RJ: Fiducial point placement and the accuracy of point-based, rigid body registration. Neurosurgery 48:810-817, 2001

31. Willems PW, Taphoorn MJ, Burger H, Berkelbach van der Sprenkel JW, Tulleken CA: Effectiveness of neuronavigation in resecting solitary intracerebral contrast-enhancing tumors: a randomized controlled trial. J Neurosurg 104:360-368, 2006

32. Wu Z, Paulsen KD, Sullivan JM Jr: Adaptive model ini- tialization and deformation for automatic segmentation of T1-weighted brain MRI data. IEEE Trans Biomed Eng 52:1128-1131, 2005

\section{Author Contributions}

Conception and design: Paulsen, Roberts, Ji. Acquisition of data: Fan, Roberts, Hartov. Analysis and interpretation of data: all authors. Drafting the article: Paulsen, Fan, Roberts. Critically revising the article: Paulsen, Fan, Roberts. Reviewed submitted version of manuscript: all authors. Approved the final version of the manuscript on behalf of all authors: Paulsen. Statistical analysis: Fan. Administrative/technical/material support: Fan, Ji, Hartov. Study supervision: Paulsen, Roberts.

\section{Correspondence}

Keith D. Paulsen, Thayer School of Engineering, Dartmouth College, 14 Engineering Dr., Hanover, NH 03755. email: keith.d.paulsen@dartmouth.edu. 